\title{
MicroRNA-21 promotes the proliferation, migration and invasion of non-small cell lung cancer A549 cells by regulating autophagy activity via AMPK/ULK1 signaling pathway
}

\author{
SHUPING $\mathrm{LI}^{1}$, XIAOFEI ZENG ${ }^{1}$, RUIDONG MA ${ }^{1}$ and LI WANG $^{2}$ \\ Departments of ${ }^{1}$ Cardiothoracic Surgery and ${ }^{2}$ Anesthesiology, The First Affiliated Hospital of Chengdu Medical College, \\ Chengdu, Sichuan 610500, P.R. China
}

Received July 30, 2017; Accepted April 24, 2018

DOI: $10.3892 /$ etm.2018.6370

\begin{abstract}
The present study investigated the expression of microRNA (miR)-21 in non-small cell lung cancer (NSCLC) tissues, its biological functions and mechanism of autophagy regulation. A total of 46 patients with NSCLC were enrolled in the present study. To measure the expression of miR-21, reverse transcription-quantitative polymerase chain reaction (RT-qPCR) was employed. NSCLC A549 cells were transfected with miR-negative control (NC), miR-21 mimics or inhibitor. The CCK-8 assay was used to investigate the proliferation of A549 cells. To study migration and invasion abilities of A549 cells, The Transwell assay was performed. In addition, to determine the expression levels of ULK1, LC3B, AMPK $\alpha, \mathrm{p}-\mathrm{AMPK} \alpha$ and $\mathrm{p} 62$ proteins, western blotting was conducted and laser confocal microscopy was performed to observe the formation of autophagosomes in A549 cells. To explore whether miR-21 regulates the biological functions of A549 cells via autophagy, an autophagy inhibitor, 3-MA, or agonist, rapamycin, were used in a rescue assay. Results indicated that miR-21 expression in NSCLC tissues was enhanced, and closely correlated with the occurrence and development of NSCLC. In vitro experiments showed that miR-21 mimics promoted the proliferation, migration and invasion of A549 cells, while miR-21 inhibitor inhibited these biological functions. Western blotting indicated that miR-21 upregulated autophagy marker LC3BII protein, but downregulated p62 protein. Laser confocal microscopy showed that miR-21 activated autophagy of A549. Rescue experiments indicated that autophagy reversed the effect of miR-21 on the proliferation, migration and invasion of A549 cells. Western blotting data suggested that autophagy-related AMPK/ULK1 signaling pathway was activated by miR-21, and interference
\end{abstract}

Correspondence to: Dr Li Wang, Department of Anesthesiology, The First Affiliated Hospital of Chengdu Medical College, 278 Baoguang Road, Chengdu, Sichuan 610500, P.R. China

E-mail: lishupingchengdu@163.com

Key words: microRNA-21, non-small cell lung cancer, autophagy or overexpression of ULK1 reversed the biological functions of miR-21. The present study demonstrated that miR-21 expression in NSCLC tissues was upregulated and positively correlated with lymphatic metastasis and clinical staging. In addition, miR-21 regulated autophagy activity of NSCLC A549 cells via AMPK/ULK1 signaling pathway, and promoted the proliferation, migration and invasion of NSCLC A549 cells.

\section{Introduction}

Lung cancer is one of the most common malignant tumors in the world (1). It is usually derived from bronchial tip membranes or glands, and accompanied by regional lymph node metastasis or hematogenous dissemination (2). Approximately 1.8 million new cases of lung cancer are reported worldwide each year, accounting for $12.9 \%$ of all new cancer cases (3). Of note, the incidence of lung cancer in men is higher than that in women. One third of new and fatal cases of lung cancer occur in China, posing a great threat to human health (4). Most lung cancer cases are malignant epithelial tumors, including non-small cell lung cancer (NSCLC; 85\%) and small cell lung cancer (15\%) (5). The clinical symptoms of NSCLC are occult, and most patients have already got metastasis when they are diagnosed. Moreover, patients with successful surgery often develop tumor resistance, leading to chemoradiotherapy failure, tumor recurrence or even death (6). It is believed that effective inhibition of lung cancer proliferation, metastasis and drug resistance will greatly improve the prognosis of patients. However, the molecular mechanisms underlying the malignant phenotype of lung cancer cells still remain unclear.

MicroRNA (miRNA or miR) molecules is a class of non-encoding small RNA molecules (18-22 nucleotides) that inhibit the translation of target genes by binding with the 3'-untranslated region (UTR) of their mRNA (7). miRNA molecules play a role as oncogenes or tumor-suppressor genes in tumor proliferation, metastasis and drug resistance, being a type of important biomarkers and therapeutic targets $(8,9)$. It is discovered that the expression of several miRNA molecules is abnormal in lung cancer, and closely related with the occurrence and development of NSCLC (6). For example, miR-30a-5p inhibits the resistance of NSCLC against paclitaxel by down-regulating the expression of 
Bcl-2 (10). In addition, miR-361-5p inhibits the proliferation and metastasis of NSCLC by targeting FOXM1 (11). miR-21 is a newly discovered miRNA molecule that is closely related with tumors (12). It is reported that miR-21 plays a role as an oncogene in NSCLC, and promotes the proliferation, invasion, metastasis and drug resistance of NSCLC $(13,14)$. However, the mechanism of action of miR-21 is still unknown.

Autophagy is a catabolic process in which cells are subjected to stress such as hypoxia, starvation, and lack of nutrients (15). Autophagy acts as a 'double-edged sword' in tumorigenesis and development $(16,17)$. On one hand, autophagy inhibits the occurrence and development of tumors by degrading abnormal components in cells or inducing non-apoptotic pathway death. On the other hand, tumor cells promote proliferation, invasion and metastasis through autophagy. Autophagy inhibitor chloroquine can greatly promote the death of tumor cells when used in combination with chemotherapy drugs (18). Studies show that miRNA molecules such as miR-181a and miR-140-5p are important in autophagy regulation $(19,20)$. It is reported that miR-21 is able to regulate the drug resistance of NSCLC (21). Therefore, it is hypothesized that miR-21 may participate in the occurrence and development of NSCLC by regulating autophagy. In the present study, we investigate the molecular mechanism by which miR-21 regulates autophagy, and try to elucidate the role of miR-21 in the development of NSCLC.

\section{Materials and methods}

Patients. A total of 46 patients with NSCLC (age range, 24-76 years; mean age, 46.7 years) who received treatments at our hospital between October 2014 and 2016 were included in the present study. NSCLC and tumor-adjacent tissues were collected as experimental and control groups, respectively. The 46 NSCLC cases included 31 cases of adenocarcinoma, 13 cases of squamous cell carcinoma, and 2 cases of adenosquamous carcinoma. Patients with lymph node metastasis (29 cases) were included into N1 subgroup, while those without lymph node metastasis (18 cases) were included into N0 subgroup. In addition, 11 patients were at stage I, 17 patients were at stage II, 13 patients were at stage III, and 6 patients were at stage IV. None of the patients received radiochemotherapy or other antineoplastic therapies. None of the patients had history of other types of tumors. All procedures were approved by the Ethics Committee of Chengdu Medical College. Written informed consents were obtained from all patients or their families.

Cells. NSCLC A549 cells were cultured in RPMI-1640 medium supplemented with $10 \%$ fetal bovine serum (FBS) under $37^{\circ} \mathrm{C}$ and $5 \% \mathrm{CO}_{2}$. When reaching 80-90\% confluency, the cells were passaged. The medium was replaced every two days. Cells with passage numbers 3-6 were used for experiments.

A549 cells were divided into miR mimics-negative control (NC) group, miR-21 mimics group, miR inhibitor-NC group, and miR-21 inhibitor group. On the day before transfection, the cells $\left(2 \times 10^{5}\right)$ in $\log$-phase growth were seeded onto 24-well plates containing antibiotics-free RPMI-1640 medium supplemented with $10 \%$ FBS. When reaching $70 \%$ confluency, $1.5 \mu 1 \mathrm{miR}$ mimics-NC (random double-stranded RNA molecule; Hanbio Biotechnology Co., Ltd., Shanghai, China),
miR-21 mimics, miR inhibitor-NC (random single-stranded RNA molecule; Hanbio Biotechnology Co., Ltd.) or miR-21 inhibitor (20 pmol $/ \mu 1$; Guangzhou RiboBio Co., Ltd., Guangzhou, China) and $1 \mu \mathrm{l}$ Lipofectamine 2000 (Thermo Fisher Scientific Inc., Waltham, MA, USA) were added into two individual vials containing $50 \mu \mathrm{l}$ Opti Memi medium, respectively. Five min later, the liquids in the two vials were mixed together before standing still for another $20 \mathrm{~min}$. Then, the mixture was added onto the cells for an incubation of $6 \mathrm{~h}$ before changing to RPMI-1640 medium supplemented with $10 \%$ FBS. The cells were cultured at $37^{\circ} \mathrm{C}$ and $5 \% \mathrm{CO}_{2}$ for $48 \mathrm{~h}$ before use.

Rescue experiments were performed in two ways. First, A549 cells $\left(2 \times 10^{5}\right)$ in miR-21 mimics and miR-21 inhibitor groups were seeded into 24-well plates containing antibiotics-free RPMI-1640 medium supplemented with $10 \%$ FBS. When reaching $70 \%$ confluency, the medium was replaced with the same medium containing $25 \mu \mathrm{M} 3-\mathrm{MA}$ and $100 \mathrm{nM}$ rapamycin and cultured at $37^{\circ} \mathrm{C}$ and $5 \% \mathrm{CO}_{2}$ for $24 \mathrm{~h}$ before rescue experiments. Second, A549 cells $\left(2 \times 10^{5}\right)$ in miR-21 mimics and miR-21 inhibitor groups were seeded into 24-well plates containing antibiotics-free RPMI-1640 medium supplemented with $10 \%$ FBS. When reaching $60 \%$ confluency, the cells were infected by $0.5 \mu \mathrm{g}$ ULK1 eukaryotic expression plasmid (Hanbio Biotechnology Co., Ltd.) or its siRNA sequence. Cells in miR-NC group were infected by $0.5 \mu \mathrm{g} \mathrm{NC}$ plasmid. After being cultured at $37^{\circ} \mathrm{C}$ and under $5 \% \mathrm{CO}_{2}$ for $6 \mathrm{~h}$, the medium was refreshed to newly made RPMI-1640 medium containing $10 \%$ FBS before cultivation for $72 \mathrm{~h}$. Then, the cells in each group were used for subsequent experiments.

Reverse transcription-quantitative polymerase chain reaction $(R T-q P C R)$. NSCLC and tumor-adjacent tissues (100 mg) were ground into powder using liquid nitrogen before addition of $1 \mathrm{ml}$ Trizol isolation reagent (Thermo Fisher Scientific, Inc.) for lysis. After lysis, total RNA was extracted using phenol chloroform method. The purity of RNA was determined by A260/A280 using ultraviolet spectrophotometry (Nanodrop ND2000, Thermo Fisher Scientific Inc.). Then, cDNA was obtained by reverse transcription using miScript II RT kit (Qiagen $\mathrm{GmbH}$, Hilden, Germany) from $1 \mu \mathrm{g}$ RNA and stored at $-20^{\circ} \mathrm{C}$.

RT-qPCR was performed using miScript SYBR-Green PCR kit (Qiagen $\mathrm{GmbH}$ ) and the reaction system was composed of $10 \mu \mathrm{l}$ RT-qPCR-mix, $0.5 \mu \mathrm{l}$ upstream primer (5'-ACAGCAGGC ACAGACAGGCAGT-3'), $0.5 \mu$ l downstream primer (universal

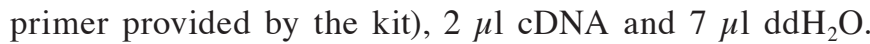
Reaction protocol was initial denaturation at $95^{\circ} \mathrm{C}$ for $10 \mathrm{~min}$, and 40 cycles of $95^{\circ} \mathrm{C}$ for $1 \mathrm{~min}$ and $60^{\circ} \mathrm{C}$ for $30 \mathrm{sec}$.

Cell Counting Kit (CCK)-8 assay. The sample cells were inoculated in 96-well plates at a density of 2,000/well. At 0, 24, 48 and 72 h, $20 \mu \mathrm{l} \mathrm{CCK-8} \mathrm{(5} \mathrm{g/l;} \mathrm{Beyotime} \mathrm{Institute}$ of Biotechnology, Haimen, China) was added onto the cells. After being incubated at $37^{\circ} \mathrm{C}$ for $2 \mathrm{~h}$, absorbance $(490 \mathrm{~nm}$ ) of each well was determined, and cell proliferation curves were plotted. Each group was tested in 3 replicate wells and the values were averaged.

Transwell assay. Matrigel chambers (Corning Incorporated, Corning, NY, USA) were used to determine the migration 
and invasion abilities of cells. Matrigel was first diluted with serum-free RPMI-1640 medium at a ratio of 1:2. In upper chamber, $50 \mu 1$ diluted Matrigel was added and kept at $37^{\circ} \mathrm{C}$ for $1 \mathrm{~h}$. Then, $1 \times 10^{5}$ cells and $200 \mu \mathrm{l}$ serum-free RPMI-1640 medium were added into the upper chamber. In the lower chamber, $500 \mu$ l RPMI-1640 medium supplemented with $10 \%$ FBS was added. After incubation for $24 \mathrm{~h}$, the cells in upper chamber were wiped by cotton swab. Then, the chamber was fixed using $4 \%$ formaldehyde for $10 \mathrm{~min}$ at room temperature, and then subjected to Giemsa's staining for $1 \mathrm{~min}$. After washing for 3 times, cells that moved to the other side of the chamber were counted under a microscope (5 fields; magnification, $\mathrm{x} 200$ ) to evaluate migration and invasion abilities.

Laser confocal microscopy. Cells in each group were seeded onto culture plates at a density of $2 \times 10^{5} /$ well. When reaching $70 \%$ confluency, Ad-GFP-RFP-LC3B was added at a ratio of $\mathrm{MOI}=20$. Seventy-two $\mathrm{h}$ later, the cells were observed under a laser confocal microscope (SP8; Leica Microsystems GmbH, Wetzlar, Germany). Green and red vesicles represented autophagy. The number of autophagy in cells was counted to evaluate autophagy activity.

Western blot analysis. Cells in each group were trypsinized and collected. Then, cold Radio-Immunoprecipitation Assay (RIPA) lysis buffer (600 $\mu \mathrm{l}$; Beyotime Institute of Biotechnology) was mixed with the samples. Then, the mixture was lysed for $30 \mathrm{~min}$ on ice, and then centrifuged at $12,000 \mathrm{rpm}$ and $4^{\circ} \mathrm{C}$ for $10 \mathrm{~min}$. Bicinchoninic acid (BCA) protein concentration determination kit (RTP7102; Real-Times Biotechnology Co., Ltd., Beijing, China) was used to determine protein concentration in the supernatant. After mixing protein samples $(6 \mu 1)$ with $5 x$ sodium dodecyl sulfate loading buffer, the mixture was denatured by boiling in water bath for $10 \mathrm{~min}$. Afterwards, 10\% sodium dodecyl sulfate-polyacrylamide gel electrophoresis $(100 \mathrm{~V})$ was performed using the samples. Then, the proteins were electro-transferred to polyvinylidene difluoride (PVDF) membranes on ice $(250 \mathrm{~mA}, 1 \mathrm{~h})$ before being blocked with $50 \mathrm{~g} / 1$ skimmed milk at room temperature for $1 \mathrm{~h}$. Afterwards, rabbit anti-human polyclonal ULK1 $(1: 1,000)$, LC3B (1:800), AMPK $\alpha(1: 1,000)$, p-AMPK $(1: 800)$ and p62 (1:800) primary antibodies and mouse anti-human GAPDH (1:400) primary antibody (Abcam, Cambridge, UK) were added onto the membranes before incubation at $4^{\circ} \mathrm{C}$ overnight. Then, the membrane was extensively washed with phosphate-buffered saline with Tween 20 (PBST) for 5 times of $5 \mathrm{~min}$, and incubated with goat anti-mouse horseradish peroxidase-conjugated secondary antibodies (1:4,000; Abcam) at room temperature for $1 \mathrm{~h}$. Subsequently, the membrane was washed with PBST for 5 times of 5 min before the membrane was developed with enhanced chemiluminescence detection kit (Sigma-Aldrich; Merck KGaA, Darmstadt, Germany) for imaging. We used Image lab v3.0 software (Bio-Rad Laboratories, Inc., Hercules, CA, USA) to acquire and analyze imaging data. The relative expression of target proteins was expressed with the ratio against GAPDH.

Statistical analysis. All results were analyzed using SPSS v17.0 statistical software (IBM Corp., Armonk, NY, USA), and all data were shown as means \pm SD. Paired Student's t-test was used to compare data between tumor-adjacent tissues and tumor tissues, while unpaired Student's t-test was performed to compare intergroup differences for lymph node metastasis and clinical staging. Multigroup measurement data were analyzed using one-way ANOVA. In case of homogeneity of variance, Least Significant Difference and Student-Newman-Keuls methods were used; in case of heterogeneity of variance, Tamhane's T2 or Dunnett's T3 method was used. $\mathrm{P}<0.05$ was considered to indicate a statistically significant difference.

\section{Results}

miR-21 expression in NSCLC tissues is enhanced, and closely correlated with the occurrence and development of NSCLC. To determine miR-21 expression in NSCLC tissues, RT-qPCR was used. The data showed that miR-21 expression in NSCLC tissues was significantly higher than that in tumor-adjacent tissues $(\mathrm{P}<0.05$; Fig. 1A). In addition, miR-21 expression in NSCLC tissues from patients with lymphatic metastasis was significantly higher than that in tissues from patients without lymphatic metastasis $(\mathrm{P}<0.05$; Fig. 1B). Moreover, miR-21 expression in NSCLC tissues from patients at stages III/IV was significantly increased than that from patients at stages I/II $(\mathrm{P}<0.05$; Fig. 1C). The result suggests that miR-21 expression in NSCLC tissues is enhanced, and closely correlated with the occurrence and development of NSCLC.

miR-21 expression promotes the proliferation of NSCLC A549 cells in vitro. To test the proliferation of A549 cells, CCK-8 assay was carried out. The data showed that absorbance of A549 cells that were transfected with miR-21 mimics was significantly enhanced than that in miR-NC group at all time points $(\mathrm{P}<0.05)$, while the absorbance of A549 cells in miR-21 inhibitor group was significantly decreased than that in miR-NC group at all time points $(\mathrm{P}<0.05$; Fig. 2$)$. The result indicates that miR-21 expression promotes the proliferation of NSCLC A549 cells.

miR-21 expression enhances the migration and invasion abilities of NSCLC A549 cells. To investigate migration and invasion abilities of A549 cells, Transwell assay was employed. The data showed that the expression of miR-21 in cells transfected with miR-21 mimics was significantly higher than that in cells transfected with miR mimics-NC $(\mathrm{P}<0.05)$, while expression of miR-21 in cells transfected with miR-21 inhibitor was significantly lower than that in cells transfected with miR inhibitor-NC $(\mathrm{P}<0.05$; Fig. 3A). The number of cells in miR-21 mimics group that crossed chamber membrane in migration assay was significantly higher than that in miR mimics-NC group $(\mathrm{P}<0.05)$. By contrast, cell count in miR-21 inhibitor group that crossed chamber membrane in migration assay was lower than that in miR inhibitor-NC group $(\mathrm{P}<0.05)$. Similarly, cell count in miR-21 mimics group that crossed chamber membrane in invasion assay was higher than that in miR mimics-NC group $(\mathrm{P}<0.05)$. In addition, the number of cells in miR-21 inhibitor group that crossed chamber membrane in invasion assay was smaller than that in miR inhibitor-NC group $(\mathrm{P}<0.05$; Fig. $3 \mathrm{~B}$ and $\mathrm{C})$. The results suggest that miR-21 expression enhances the migration and invasion abilities of NSCLC A549 cells. 

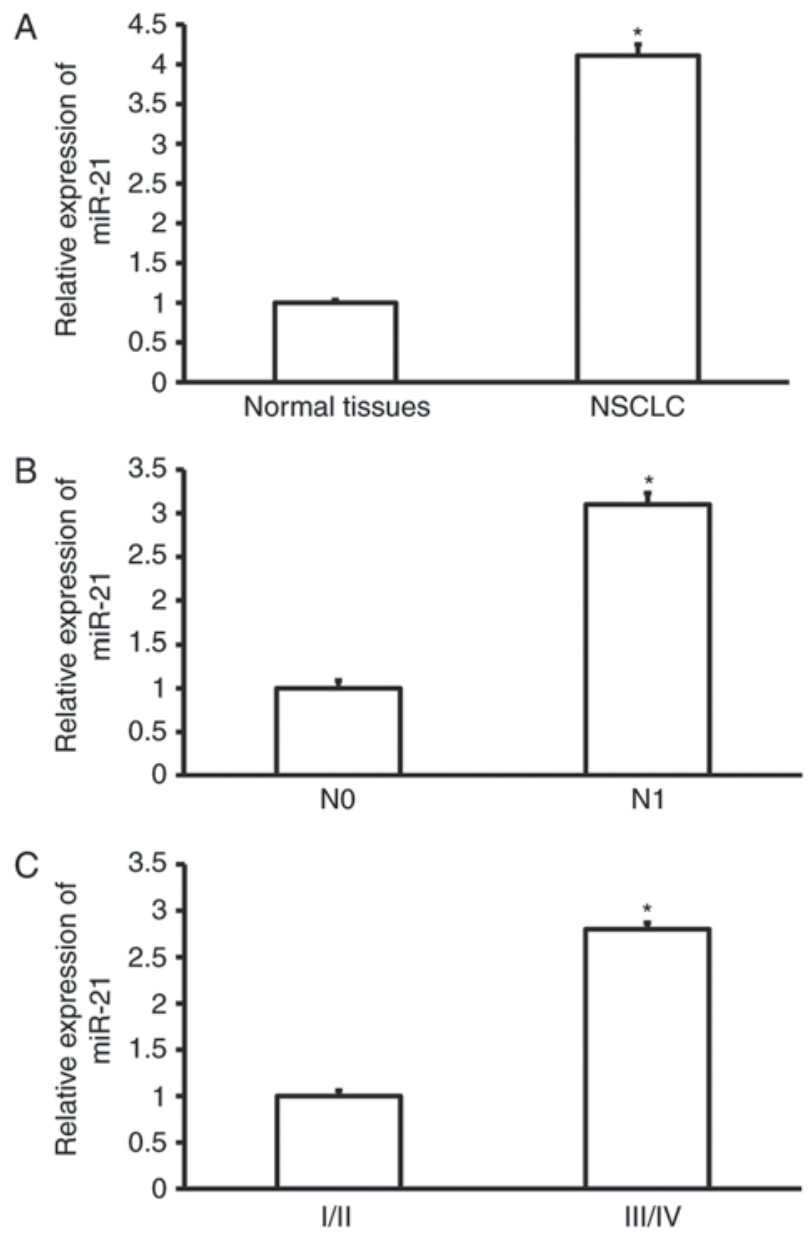

Figure 1. Expression of miR-21 in NSCLC tissues. (A) Expression of miR-21 in tumor-adjacent normal tissues and NSCLC tissues. $\mathrm{P}<0.05$ compared with normal tissues. (B) Expression of miR-21 in NSCLC tissues from NSCLC patients without (N0) and with (N1) lymphatic metastasis. " $\mathrm{P}<0.05$ compared with N0 group. (C) Expression of miR-21 in NSCLC tissues from patients at different clinical stages. ${ }^{*} \mathrm{P}<0.05$ compared with patients at stages I/II. NSCLC, non-small cell lung cancer.

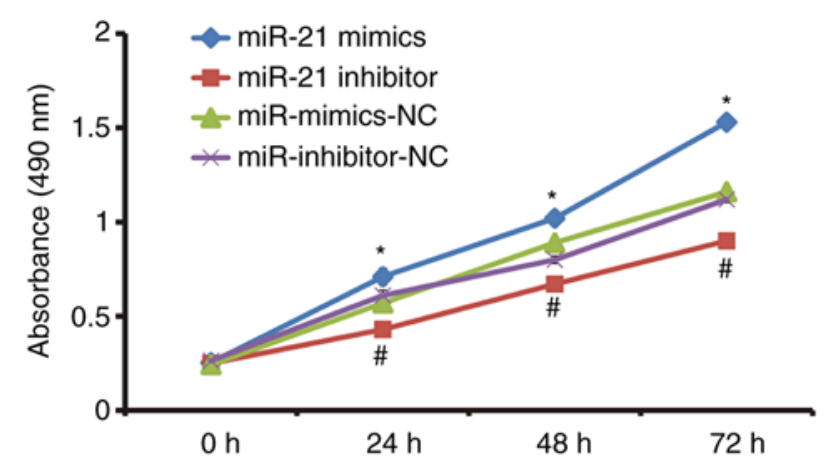

Figure 2. Effect of miR-21 on the proliferation of NSCLC A549 cells. The cells were transfected with miR-NC, miR-21 mimics or miR-21 inhibitor. CCK- 8 assay was used to determine the proliferation of cells. ${ }^{*} \mathrm{P}<0.05$ compared with miR-mimics-NC group at respective time points; ${ }^{*} \mathrm{P}<0.05$ compared with miR-inhibitor-NC group. NSCLC, non-small cell lung cancer; $\mathrm{NC}$, negative control.

miR-21 regulates autophagy activities of NSCLC A549 cells. To test the expression of autophagy-related proteins in A549 cells, western blotting was performed. Quantification of western blots showed that LC3B-II protein expression in
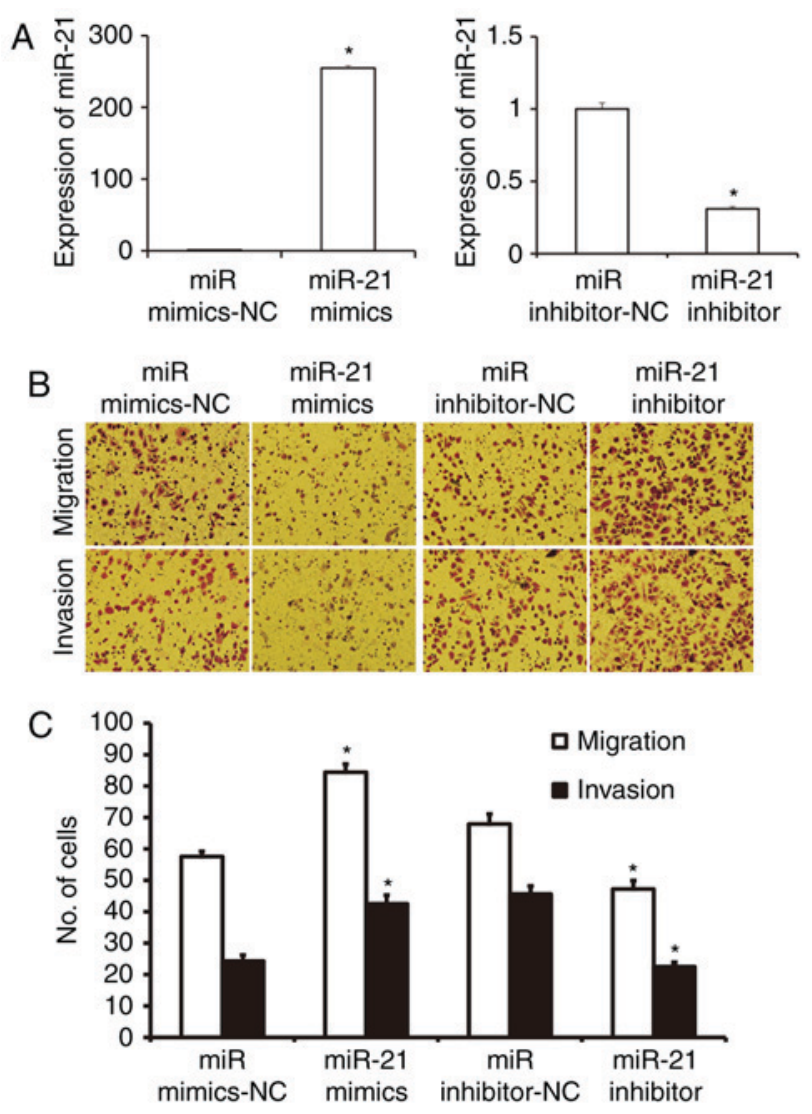

Figure 3. Effect of miR-21 on the migration and invasion of NSCLC A549 cells. The cells were transfected with miR mimics-NC, miR-21 mimics, miR inhibitor-NC, or miR-21 inhibitor. (A) Expression of miR-21 in cells transfected with miR mimics-NC, miR-21 mimics, miR inhibitor-NC, or miR-21 inhibitor. " $\mathrm{P}<0.05$ compared with NC group. (B) Images of migrated and invasion cells in indicated groups. Magnification, x200. (C) The numbers of migrated and invasion cells in each group. Transwell assay was used to determine the migration and invasion of A549 cells. " $\mathrm{P}<0.05$ compared with miR-NC group. NSCLC, non-small cell lung cancer; NC, negative control.

miR-21 mimics group was significantly higher than that in miR mimics-NC group $(\mathrm{P}<0.05)$, while p62 protein expression in miR-21 mimics group was significantly lower than that in miR mimics-NC group $(\mathrm{P}<0.05)$. In addition, LC3B-II protein expression in miR-21 inhibitor group was significantly lower than that in miR inhibitor-NC group $(\mathrm{P}<0.05)$, while $\mathrm{p} 62$ protein expression in miR-21 inhibitor group was significantly higher than that in miR inhibitor-NC group ( $\mathrm{P}<0.05$; Fig. 4$)$. The result suggests that miR-21 regulates autophagy activities of NSCLC A549 cells.

miR-21 expression facilitates the autophagy of NSCLC A549 cells. To observe the formation of autophagosomes in A549 cells, laser confocal microscopy was performed. The data showed that the number of autophagosomes in A549 cells of miR-21 mimics group was significantly higher than that in miR mimics-NC group $(\mathrm{P}<0.05)$, while that of miR-21 inhibitor group was significantly lower than that in miR mimics-NC group $(\mathrm{P}<0.05$; Fig. 5). The result indicates that miR-21 expression facilitates the autophagy of NSCLC A549 cells.

miR-21 promotes the proliferation, migration and invasion of NSCLC A549 cells by regulating autophagy. To test whether 


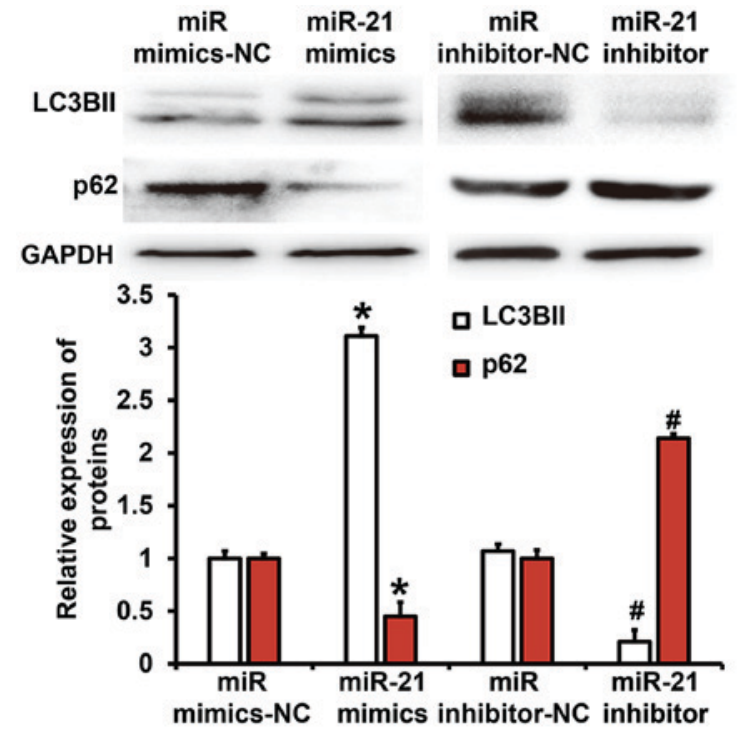

Figure 4. Expression of LC3BII and p62 proteins in NSCLC A549 cells. The cells were transfected with miR mimics-NC, miR-21 mimics, miR inhibitor-NC or miR-21 inhibitor before determination of LC3BII and $\mathrm{p} 62$ protein expression by western blotting. ${ }^{*} \mathrm{P}<0.05$ compared with miR mimics-NC group; ${ }^{\#} \mathrm{P}<0.05$ compared with miR inhibitor-NC group. NSCLC, non-small cell lung cancer; $\mathrm{NC}$, negative control.

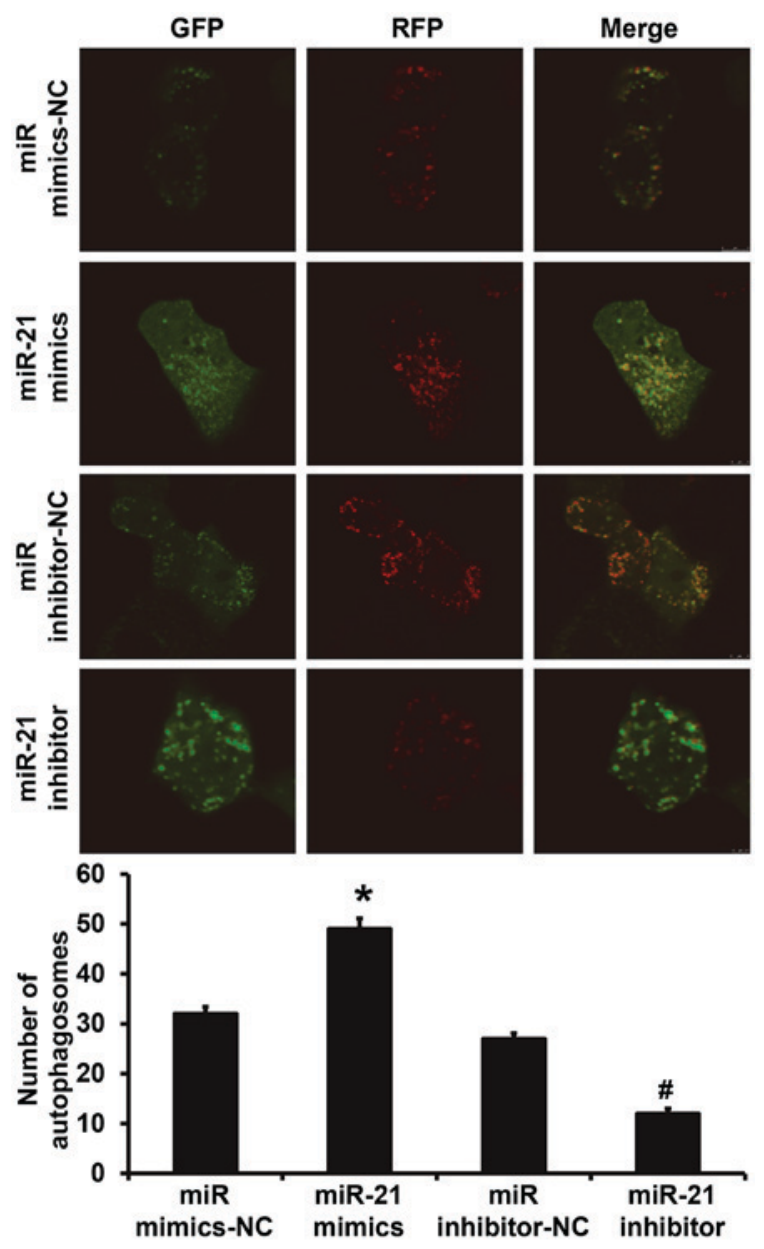

Figure 5. Effect of miR-21 expression on the number of autophagosomes in NSCLC A549 cells. To observe the formation of autophagosomes in A549 cells, laser confocal microscopy was performed. Magnification, x200. Green and red vesicles represented autophagy. "P $<0.05$ compared with miR mimics-NC group; ${ }^{\prime \prime} \mathrm{P}<0.05$ compared with miR inhibitor-NC group. NSCLC, non-small cell lung cancer; NC, negative control.
miR-21 regulates the biological functions of A549 cells via autophagy, we used autophagy inhibitor 3-MA or agonist rapamycin. CCK-8 assay showed that treatment with 3-MA decreased the elevated proliferation of A549 cells that was induced by miR-21 mimics transfection to a level similar to miR-NC group $(\mathrm{P}<0.05$; Fig. 6A). In addition, treatment with rapamycin increased the reduced proliferation of A549 cells that was induced by miR-21 inhibitor transfection to a level similar to miR-NC group $(\mathrm{P}<0.05$; Fig. $6 \mathrm{~B})$. Transwell assay showed that treatment with 3-MA decreased the elevated migration and invasion abilities of A549 cells that were induced by miR-21 mimics transfection to a level similar to miR-NC group $(\mathrm{P}<0.05$; Fig. 6C), while treatment with rapamycin increased the reduced migration and invasion abilities of A549 cells that were induced by miR-21 inhibitor transfection to a level similar to miR-NC group ( $\mathrm{P}<0.05$; Fig. 6D). The results suggest that miR-21 promotes the proliferation, migration and invasion of NSCLC A549 cells by regulating autophagy.

miR-21 regulates the autophagy of NSCLC A549 cells via AMPK/ULK1 signaling pathway. To further investigate the molecular mechanism by which miR-21 regulates autophagy, we predicted the target gene of miR-21 using TargetScan (http://www.targetscan.org/), but failed to discover any autophagy-related gene. Therefore, we examined autophagy-related signaling pathways using western blotting. The data showed that miR-21 up-regulated AMPK $\alpha$ subunit phosphorylation level and ULK1 expression (Fig. 7A). In addition, overexpression of miR-21 significantly increased the expression of LC3BII protein $(\mathrm{P}<0.05)$, but silencing of ULK1 by its siRNA significantly reduced the expression of LC3BII protein $(\mathrm{P}<0.05)$. By contrast, miR-21 inhibitor significantly decreased the expression of LC3BII protein $(\mathrm{P}<0.05)$, but overexpression of ULK1 significantly enhanced the expression of LC3BII protein $(\mathrm{P}<0.05$; Fig. 7B). These results indicate that miR-21 regulates the autophagy of NSCLC A549 cells via AMPK/ULK1 signaling pathway.

\section{Discussion}

NSCLC patients often lose their best surgical chances because of extensive metastasis. Radiotherapy and chemotherapy can easily induce drug resistance of tumor cells and cause tumor recurrence and metastasis, leading to death of patients (22). Studies show that the development of NSCLC is a complex process involving multiple genes, multiple factors and multiple steps, such as gene mutation, abnormal expression, immunosuppression and tumor stem cells $(23,24)$. Of note, miRNA molecules are involved in the regulation of various malignant phenotypes of NSCLC, and have become potential tumor therapy targets (25). In the present study, we discover that miR-21 expression in NSCLC tissues is significantly elevated, and positively correlated with lymphatic metastasis and clinical staging, suggesting that miR-21 may play a role as an oncogene. In vitro experiments demonstrate that miR-21 regulates autophagy of NSCLC cells via AMPK/ULK1 signaling pathway, and facilitates the proliferation, migration and invasion of NSCLC cells.

It is reported that miR-21 plays important roles in the occurrence and development of various malignant tumors. 

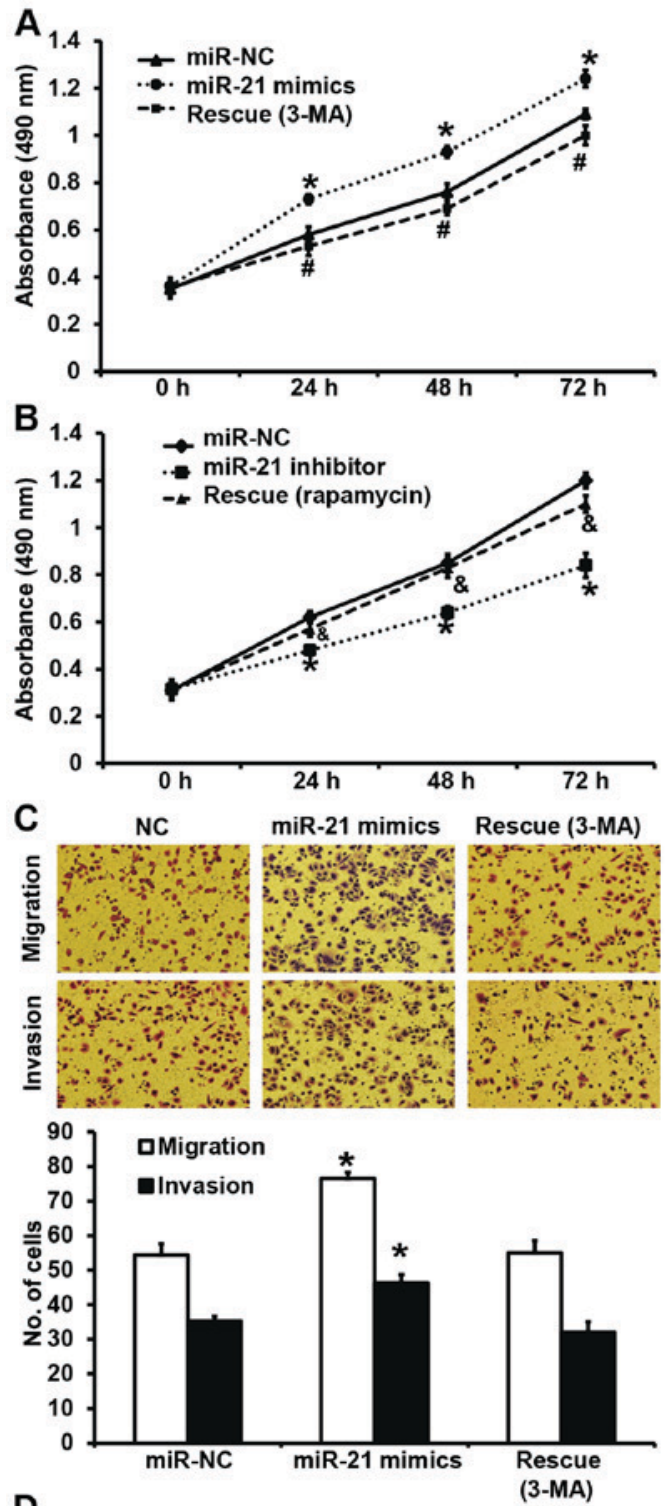

D
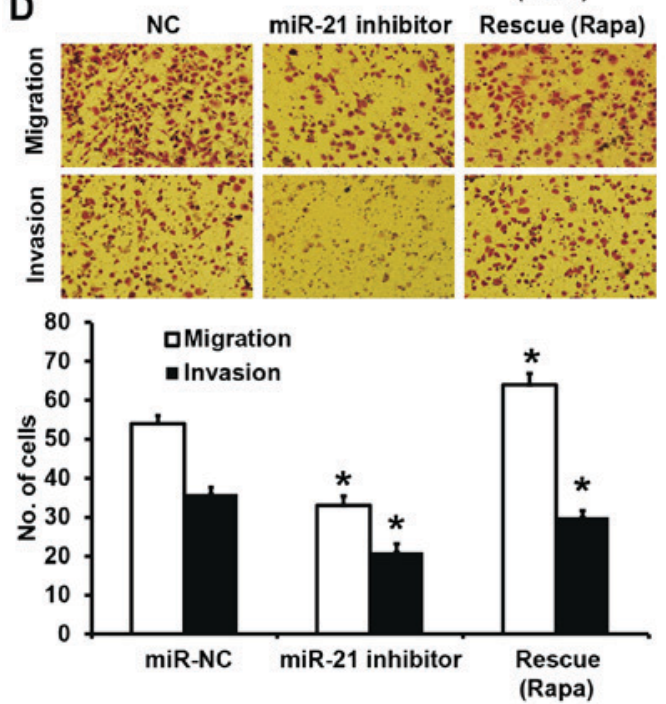

Figure 6. Effects of autophagy inhibitor or agonist on biological functions of A549 cells. (A and B) Proliferation of A549 cells after treatment with (A) autophagy inhibitor 3-MA or (B) autophagy agonist rapamycin. ${ }^{*} \mathrm{P}<0.05$ compared with miR-NC group; ${ }^{*} \mathrm{P}<0.05$ compared with miR-21 mimics group; ${ }^{\mathrm{P}} \mathrm{P}<0.05$ compared with miR-21 inhibitor group. (C and D) Migration and invasion of A549 cells after treatment with (C) autophagy inhibitor 3-MA or (D) autophagy agonist rapamycin. Magnification, $\mathrm{x} 200 .{ }^{*} \mathrm{P}<0.05$ compared with miR-NC group. $\mathrm{NC}$, negative control.
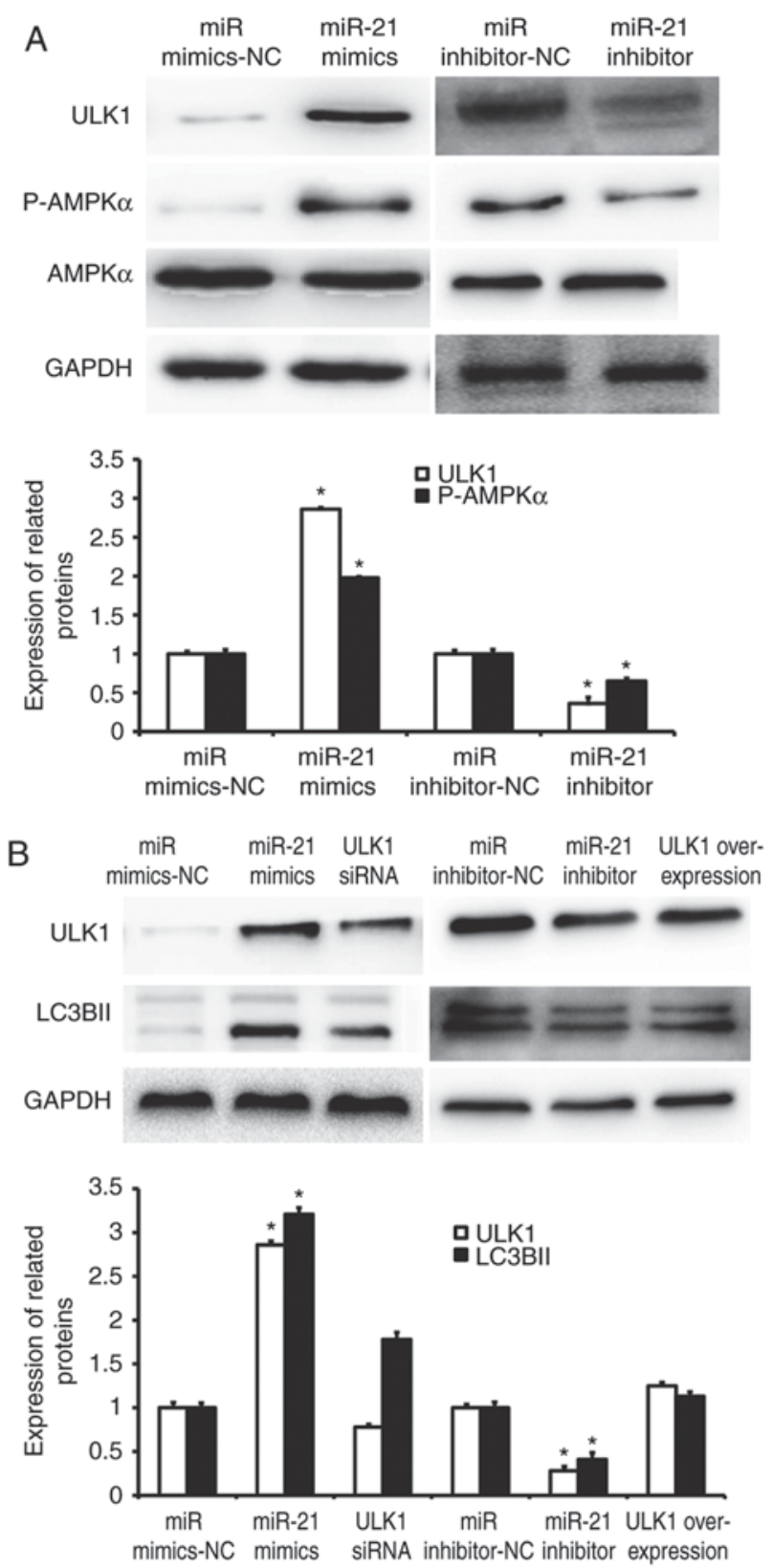

Figure 7. Regulation of autophagy of NSCLC cells by miR-21 via AMPK/ULK1 signaling pathway. (A) Activation of AMPK/ULK1 signaling pathway by miR-21. (B) ULK1 rescues the regulation of miR-21 on autophagy of A549 cells. Western blotting was used to determine the expression of ULK1, P-AMPK $\alpha$, AMPK $\alpha$, and LC3BII. The data were derived from the same membrane, and the membrane was stripped and re-probed. ${ }^{*} \mathrm{P}<0.05$ compared with miR mimics-NC group; ${ }^{~} \mathrm{P}<0.05$ compared with miR inhibitor-NC group. NC, negative control

Expression of miR-21 is up-regulated in various human tumor tissues and cells, such as brain glioma, ovarian cancer, bladder cancer, prostate cancer, lung cancer, breast cancer, thyroid cancer, esophageal cancer, liver cancer, bile duct cancer, pancreatic cancer, colorectal cancer, gastric cancer, and B cell lymphoma. miR-21 participates in the proliferation, migration, invasion, differentiation and apoptosis of tumor cells $(26,27)$. In addition, miR-21 is involved in the regulation of multiple downstream target genes such as PTEN, PDCD4, RECK, and TIMP-3 $(21,28)$. Although there are many reports about the regulation of tumor cell proliferation, migration and invasion 
by $\mathrm{miR}-21$, there is no literature report on whether miR-21 regulates autophagy activity in NSCLC. Our study shows that miR-21 is up-regulated in NSCLC, and is closely related to TNM staging and lymph node metastasis. In addition, miR-21 activates the autophagy of A549 cells by up-regulating LC3BII protein expression, down-regulating p62 protein expression, and increasing the number of autophagosomes in A549 cells. Of note, rescue experiments show that autophagy inhibitor or agonist can rescue the changes in biological functions of A549 cells caused by up-regulation or down-regulation of miR-21. This also suggests that miR-21 can exert its biological function of oncogene by activating autophagy.

Autophagy is closely related to the occurrence and development of tumors. It participates in the proliferation, metastasis and invasion of tumors mainly through three ways. First, autophagy provides energy for tumors and promotes tumor cell survival. Second, autophagy inhibits tumor cell apoptosis. Third, autophagy enhances the tolerance of tumor cells to radiotherapy and chemotherapy. For example, Pursiheimo et al (29) report that hypoxia induces autophagy and increases cell survival rate. Moon et al (30), show that autophagy protects ovarian cancer cells against metformin-induced apoptosis. Regulating autophagy activity has also become a new strategy for cancer therapy. AMPK signaling pathway is one of the key factors for intracellular and extracellular energy integration, and is closely related to autophagy. It is reported that AMPK increases cell survival rate by promoting autophagy via the up-regulation of ULK1 (31). In the present study, our data show that the phosphorylation level of AMPK $\alpha$ subunit is up-regulated, and the expression of ULK1, a key gene of autophagy, is increased, suggesting that miR-21 may regulate autophagy via ULK1. Consistently, siRNA interference and overexpression of ULK1 restore autophagy activity of A549 cells. The reason why we suspect LC3BII and ULK1 p-AMPK-alpha and LC3BII expression are different among the miR mimics-NC and miR inhibitor-NC groups is that miR mimics-NC is double-stranded and miR inhibitor-NC is single-stranded. The response of autophagy to the two sequences may be different.

The limitations of the present study include small sample size, and the subsequent lack of correlation analysis between miR-21 expression and N2/N3 lymph node status. In addition, the clinical correlation of miR-21 to NSCLC remains to be further verified.In future studies, we will use specific autophagy inhibitors to further verify the regulatory role of miR-21 on autophagy. In conclusion, the present study demonstrates that miR-21 is highly expressed in NSCLC tissues, and closely related to the occurrence and development of NSCLC. miR-21 can also activate autophagy via the AMPK/ULK1 pathway and play a role as oncogene. Therefore, miR-21 is a potential therapeutic target for NSCLC.

\section{Acknowledgements}

Not applicable.

\section{Funding}

This work was supported by the Project of Sichuan Provincial Health Bureau (grant no. 17PJ497).

\section{Availability of data and materials}

The analyzed data sets generated during the study are available from the corresponding author on reasonable request.

\section{Authors' contributions}

SL and LW collaborated to design the study. SL, XZ and RM were responsible for all experiments. SL and LW analyzed the data. All authors collaborated to interpret results and develop the manuscript. The final version of the manuscript has been read and approved by all authors.

\section{Ethical approval and consent to participate}

All procedures performed in the current study were approved by the Ethics Committee of Chengdu Medical College. Written informed consent was obtained from all patients or their families.

\section{Consent for publication}

Not applicable.

\section{Competing interests}

The authors declare that they have no competing interests.

\section{References}

1. Schneider G, Sellers ZP, Bujko K, Kakar SS, Kucia M and Ratajczak MZ: Novel pleiotropic effects of bioactive phospholipids in human lung cancer metastasis. Oncotarget 8: 58247-58263, 2017.

2. Choi EJ, Jung BJ, Lee SH, Yoo HS, Shin EA, Ko HJ, Chang S, Kim SY and Jeon SM: A clinical drug library screen identifies clobetasol propionate as an NRF2 inhibitor with potential therapeutic efficacy in KEAP1 mutant lung cancer. Oncogene 36: 5285-5295, 2017.

3. Tian Y, Liu Q, Wu K, Chu Q, Chen Y and Wu K: Meta-analysis comparing the efficacy of nedaplatin-based regimens between squamous cell and non-squamous cell lung cancers. Oncotarget 8: 62330-62338, 2017.

4. Tong YH, Zhang B, Yan YY, Fan Y, Yu JW, Kong SS, Zhang D, Fang L, Su D and Lin NM: Dual-negative expression of Nrf2 and NQO1 predicts superior outcomes in patients with non-small cell lung cancer. Oncotarget 8: 45750-45758, 2017.

5. Xu W, Wu B, Fu L, Chen J, Wang Z, Huang F, Chen J, Zhang M, Zhang Z, Lin J, et al: Comparison of three different methods for the detection of circulating tumor cells in mice with lung metastasis. Oncol Rep 37: 3219-3226, 2017.

6. Zhang Y, Sui J, Shen X, Li C, Yao W, Hong W, Peng H, Pu Y, Y in L and Liang G: Differential expression profiles of microRNAs as potential biomarkers for the early diagnosis of lung cancer. Oncol Rep 37: 3543-3553, 2017.

7. Icli $\mathrm{B}$ and Feinberg MW: MicroRNAs in dysfunctional adipose tissue: Cardiovascular implications. Cardiovasc Res 113: 1024-1034, 2017

8. Avellini C,Licini C, Lazzarini R, Gesuita R, Guerra E, Tossetta G, Castellucci C, Giannubilo SR, Procopio A, Alberti S, et al: The trophoblast cell surface antigen 2 and miR-125b axis in urothelial bladder cancer. Oncotarget 8: 58642-58653, 2017.

9. Xiang Y, Liao XH, Yu CX, Yao A, Qin H, Li JP, Hu P, Li H, Guo W, Gu CJ and Zhang TC: MiR-93-5p inhibits the EMT of breast cancer cells via targeting MKL-1 and STAT3. Exp Cell Res 357: 135-144, 2017.

10. Xu X, Jin S, Ma Y, Fan Z, Yan Z, Li W, Song Q, You W, Lyu Z, Song Y, et al: miR-30a-5p enhances paclitaxel sensitivity in non-small cell lung cancer through targeting BCL-2 expression. J Mol Med (Berl) 95: 861-871, 2017. 
11. Hou XW, Sun X, Yu Y, Zhao HM, Yang ZJ, Wang X and Cao XC: miR-361-5p suppresses lung cancer cell lines progression by targeting FOXM1. Neoplasma 64: 526-534, 2017.

12. Arias Sosa LA, Cuspoca Orduz AF and Bernal Gómez BM: Deregulation of microRNAs in gastric cancer: Up regulation by miR-21 and miR-106. Rev Gastroenterol Peru 37: 65-70, 2017 (In Spanish).

13. Markou A, Zavridou M and Lianidou ES: miRNA-21 as a novel therapeutic target in lung cancer. Lung Cancer (Auckl) 7: 19-27, 2016.

14. Dong J, Zhang Z, Gu T, Xu SF, Dong LX, Li X, Fu BH and Fu ZZ: The role of microRNA-21 in predicting brain metastases from non-small cell lung cancer. Onco Targets Ther 10: 185-194, 2016

15. Horibe A, Eid N, Ito Y, Hamaoka H, Tanaka Y and Kondo Y: Upregulated autophagy in sertoli cells of ethanol-treated rats is associated with induction of inducible nitric oxide synthase (iNOS), androgen receptor suppression and germ cell apoptosis. Int J Mol Sci 18: pii: E1061, 2017.

16. Krause GC, Lima KG, Dias HB, da Silva EFG, Haute GV, Basso BS, Gassen RB, Marczak ES, Nunes RSB and de Oliveira JR: Liraglutide, a glucagon-like peptide-1 analog, induce autophagy and senescence in HepG2 cells. Eur $\mathrm{J}$ Pharmacol 809: 32-41, 2017.

17. Zheng Z, Xu L, Zhang S, Li W, Tou F, He Q, Rao J and Shen Q: Peiminine inhibits colorectal cancer cell proliferation by inducing apoptosis and autophagy and modulating key metabolic pathways. Oncotarget 8: 47619-47631, 2017.

18. Dai R, Zhang S, Duan W, Wei R, Chen H, Cai W, Yang L and Wang Q: Enhanced autophagy contributes to protective effects of GM1 Ganglioside Against A $\beta 1-42$-induced neurotoxicity and cognitive deficits. Neurochem Res 42: 2417-2426, 2017.

19. Lin Y, Zhao J, Wang H, Cao J and Nie Y: miR-181a modulates proliferation, migration and autophagy in AGS gastric cancer cells and downregulates MTMR3. Mol Med Rep 15: 2451-2456, 2017.

20. Wei R, Cao G, Deng Z, Su J and Cai L: miR-140-5p attenuates chemotherapeutic drug-induced cell death by regulating autophagy through inositol 1,4,5-trisphosphate kinase 2(IP3k2) in human osteosarcoma cells. Biosci Rep 36: pii: e00392, 1016.

21. Jiang LP, He CY and Zhu ZT: Role of microRNA-21 in radiosensitivity in non-small cell lung cancer cells by targeting PDCD4 gene. Oncotarget 8: 23675-23689, 2017.
22. Yuan C, Zheng Y, Zhang B, Shao L, Liu Y, Tian T, Gu X, Li X and Fan K: Thymosin $\alpha 1$ promotes the activation of myeloid-derived suppressor cells in a Lewis lung cancer model by upregulating Arginase 1. Biochem Biophys Res Commun 464: 249-255, 2015.

23. Puspawati NLPD, Sitorus R and Herawati T: Hand-held Fan airflow stimulation relieves dyspnea in lung cancer patients. Asia Pac J Oncol Nurs 4: 162-167, 2017.

24. Deng ZM, Liu L, Qiu WH, Zhang YQ, Zhong HY, Liao P and $\mathrm{Wu} \mathrm{YH}$ : Analysis of genomic variation in lung adenocarcinoma patients revealed the critical role of PI3K complex. PeerJ 5: e3216, 2017.

25. Duan B, Guo T, Sun H, Cai R, Rui Q and Xi Z: miR-205 as a biological marker in non-small cell lung cancer. Biomed Pharmacother 91: 823-830, 2017.

26. Zhang L, Yao J, Li W and Zhang C: Micro-RNA-21 regulates cancer-associated fibroblast-mediated frug resistance in pancreatic cancer. Oncol Res, May 5, 2017 (Epub ahead of print).

27. Feng Y, Zou W, Hu C, Li G, Zhou S, He Y, Ma F, Deng C and Sun L: Modulation of CASC2/miR-21/PTEN pathway sensitizes cervical cancer to cisplatin. Arch Biochem Biophys 623-624: 20-30, 2017.

28. Shao YY, Zhang TL, Wu LX, Zou HC, Li S, Huang J and Zhou HH: AKT axis, miR-21, and RECK play pivotal roles in dihydroartemisinin killing malignant glioma cells. Int J Mol Sci 18: pii: E350, 2017.

29. Pursiheimo JP, Rantanen K, Heikkinen PT, Johansen T and Jaakkola PM: Hypoxia-activated autophagy accelerates degradation of SQSTM1/p62. Oncogene 28: 334-344, 2009.

30. Moon HS, Kim B, Gwak H, Suh DH and Song YS: Autophagy and protein kinase RNA-like endoplasmic reticulum kinase (PERK)/eukaryotic initiation factor 2 alpha kinase (eIF2 $\alpha$ ) pathway protect ovarian cancer cells from metformin-induced apoptosis. Mol Carcinog 55: 346-356, 2016.

31. Hong-Brown LQ, Brown CR, Navaratnarajah $\mathrm{M}$ and Lang $\mathrm{CH}$ : FoxO1-AMPK-ULK1 regulates ethanol-induced autophagy in muscle by enhanced ATG14 association with the BECN1-PIK3C3 complex. Alcohol Clin Exp Res 41: 895-910, 2017. 